\title{
Correction to: $\left[\mathrm{Pt}\left(0,0^{\prime}\right.\right.$-acac) $(\gamma$-acac)(DMS)]: Alternative Strategies to Overcome Cisplatin-Induced Side Effects and Resistance in T98G Glioma Cells
}

\author{
Valentina Astesana ${ }^{1}$. Pawan Faris ${ }^{2,3}$. Beatrice Ferrari ${ }^{1}$ - Stella Siciliani ${ }^{1}$ - Dmitry Lim ${ }^{4}$ - Marco Biggiogera ${ }^{1}$. \\ Sandra Angelica De Pascali ${ }^{5}$. Francesco Paolo Fanizzi ${ }^{5}$. Elisa Roda ${ }^{1,6}$. Francesco Moccia ${ }^{2} \cdot$ Maria Grazia Bottone $^{1}($ (i)
}

Published online: 13 June 2020

(c) Springer Science+Business Media, LLC, part of Springer Nature 2020

\section{Correction to: Cellular and Molecular Neurobiology https://doi.org/10.1007/s10571-020-00873-8}

The original version of the article unfortunately contained a typo in co-author name.

The author name should be Francesco Paolo Fanizzi instead it was published incorrectly as Franco Paolo Fanizzi.

The original article has been corrected.

Publisher's Note Springer Nature remains neutral with regard to jurisdictional claims in published maps and institutional affiliations.

The original article can be found online at https://doi.org/10.1007/ s10571-020-00873-8

Maria Grazia Bottone

bottone@unipv.it

1 Laboratory of Cell Biology and Neurobiology, Department of Biology and Biotechnology "L. Spallanzani”, University of Pavia, via Ferrata 9, 27100 Pavia, Italy

2 Laboratory of General Physiology, Department of Biology and Biotechnology 'L. Spallanzani', University of Pavia, 27100 Pavia, Italy

3 Department of Biology, Cihan University-Erbil, Erbil 44001, Iraq

4 Department of Pharmaceutical Sciences, Università degli Studi del Piemonte Orientale, 28100 Novara, Italy

5 General and Inorganic Chemistry Laboratory, Department of Biological and Environmental Sciences and Technologies (DiSTeBA), University of Salento, Lecce, Italy

6 Laboratory of Clinical \& Experimental Toxicology, Pavia Poison Centre, National Toxicology Information Centre, Toxicology Unit, ICS Maugeri Spa, IRCCS Pavia, Pavia, Italy 